\title{
3-dimensional analysis about the effects of aging and risk factors on changes in oral environment
}

\author{
Yong Yoon', Yong-Gun Kim', Sang-Kyu Lee ${ }^{2 *}$, Jae-Mok Lee ${ }^{1 *}$ \\ 'Department of Periodontics, College of Dentistry, Kyungpook National University, Daegu, Republic of Korea \\ ${ }^{2}$ College of Pharmacy and Research Institute of Pharmaceutical Sciences, Kyungpook National University, Daegu, Republic of Korea
}

\begin{abstract}
PURPOSE. The purpose of this study is to investigate the effects of aging and various risk factors on the oral environment and to analyze them in 3-dimensions. MATERIALS AND METHODS. A total of 800 patients were enrolled in this study, and subjects were divided into 4 groups by age-under 55, 56 - 65, 66 - 75, and over 76. Based on their most recent visit, the number of crowns, bridges, implants, and the remaining natural teeth were recorded. Smoking habits, along with presence of diabetes and hypertension, were surveyed, as risk factors were also set as a variable. Comparisons among the groups or within the groups were performed by independent t-test, and one-way and two-way ANOVA. Kruskal-Wallis test and Mann-Whitney $U$ test were used for analysis. It was assumed to be statistically significant when $P$ value is below .05. RESULTS. Changes in the number of crowns, bridges, implants, and the remaining natural teeth by age were statistically significant. When we examined the effect of risk factors on the change of variables with age, hypertension was found to affect the number of bridges. Diabetes and smoking were found to affect the number of the remaining natural teeth. The other variables were not statistically significant. CONCLUSION. Aging is considered to be an important variable affecting the change of oral environment. Among the risk factors, the presence of smoking habit and diabetes is thought to have a great influence on the change of the number of the remaining natural teeth. [J Adv Prosthodont 2019;11:75-80]
\end{abstract}

KEYWORDS: Aging; Risk factors; Hypertension; Diabetes; Smoking

\section{INTRODUCTION}

Chronic periodontitis is an inflammatory disease of the periodontal tissue around the teeth that is caused by bacteria. As a result, gingival recession, and periodontal ligament and alveolar bones are destroyed. In addition to bacteria,

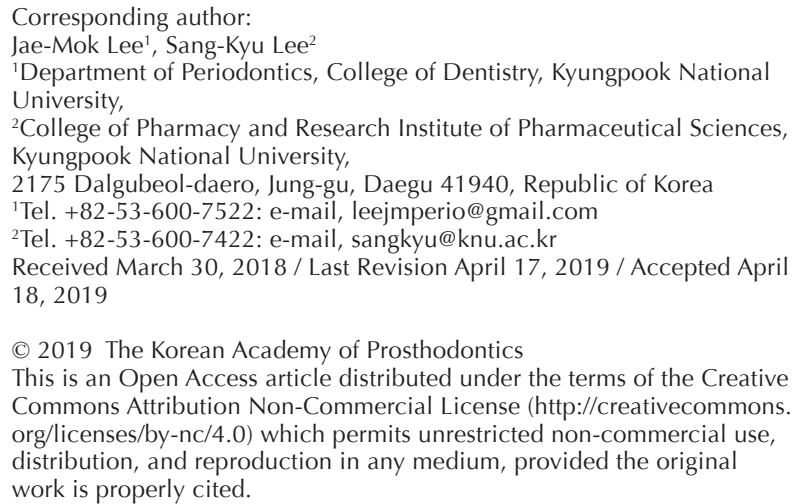

factors affecting periodontal disease include aging, systemic factors such as diabetes and hypertension, and oral habits such as smoking.

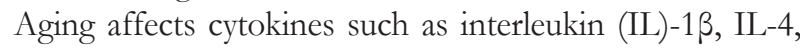
IL-6, IL-8 and bone destruction factors such as receptor activator of nuclear factor- $x \mathrm{~B}$ ligand (RANKL) and osteoprotegerin (OPG) have been actively studied. ${ }^{1-3}$ Banatti et al..$^{3}$ investigated changes in cell proliferation, viability, and total secreted protein in periodontal ligament (PDL) cells, where aging plays an important role in periodontal tissue destruction. As a result, data suggested that aging decreased the ability of PDL cell to proliferate and modulated the expression of important inflammatory and bone-related genes in periodontal apparatus, favoring a proinflammatory and antiresorptive profile.

Increased awareness of health, along with the advancement of preventive dentistry, has resulted in a decrease in tooth loss across all age groups. Careful consideration should be given to the effect that this trend has on tooth maintenance. Increased life expectancy and higher standards of 
health can change the needs of the elderly towards periodontal treatment, and lead to a substantial increase in periodontal care practices. ${ }^{4,5}$ Therefore, it is important to understand the effect of aging on periodontal tissue.

Normal teeth and their surrounding tissue undergo significant histological and clinical changes with age. Such changes are both morphological and functional and are associated with changes in the oral environment. The periodontium of the elderly is characterized as follows: atrophy of the oral mucosa, a tendency toward decreased stippling, thinning of the epithelium, reduced elastic properties of connective tissue, and decreased microvascularity as a result of reduced blood supply. Clinically, gingiva of the elderly show recession and migration to apex, along with decreased elasticity and consequent increased sensitivity to external factors. ${ }^{6}$

The association between aging and alveolar bone resorption (ABL) is difficult to assess due to individual hygiene and immunological factors and differences in the chronic nature of periodontal disease. However, many animal studies have shown that there is a correlation between aging and ABL.?

Aging is known to negatively affect steps in the healing process such as hemostasis, reduction of inflammation, cell proliferation and migration, and secretion of extracellular matrix. Clinically, aging is known to delay the recovery of gingival tissue in wound healing. In this respect, many research papers in periodontology have reported that the aging process promotes degenerative changes and contributes to the destruction of periodontal tissue through atherosclerosis and consequent decrease in blood supply. In addition, aging results in wider PDL space, decreased number of periodontal fibers and smaller cells. Mineral density of alveolar bone decreases, and less bone remodeling occurs $^{8}$

In a classic experimental study on gingivitis, subjects were kept in a plaque-free and inflammation-free state through professional management for three weeks. Then, professional management was ceased and gingivitis was induced in young and elderly subjects. Results showed more severe inflammation in older individuals in both human and dog models. In order to more accurately differentiate the effect of age, other studies have eliminated disturbance to focus on obtaining a clearer view of whether age is a risk factor in gingivitis. A "risk factor" can be defined as traits, behaviors, and exposure to certain factors that increases the likelihood of a disease. Some risk factors, such as smoking or improved oral hygiene, can be modified to decrease the risk of initiation or progression of a disease, while risk factors like genetics cannot be changed. The conclusions of the previous studies agree consistently that the effect of age is either non-existent or clinically insignificant. In fact, the odds of age causing periodontitis were only 1.24 , whereas the odds were 20.52 for poor oral hygiene. Some studies report that smoking has a greater effect than age. ${ }^{9}$

Though various studies exist on the effect of age on oral health, there is still a lack of 3-dimensional studies that incorporate "risk factors" into the question. Thus, this study aims not only to investigate changes in oral conditions related to age, but also to find their correlation with risk factors such as smoking, diabetes, and hypertension.

\section{MATERIALS AND METHODS}

800 patients who visited the Kyungpook National University Dental Hospital between April 2011 and May 2014 were analyzed in this study. Subjects were composed of patients consistently receiving maintenance periodontal treatment for 5 years or longer. Exclusion criteria included patients who were not consistent in receiving treatment, and patients who had to receive periodontal treatment again due to extremely poor oral hygiene.

Subjects were divided into 4 groups by age-under 55, 56 65, $66-75$, and over 76. Based on their most recent visit, a number of crowns, bridges, implants, and the remaining natural teeth were recorded. 200 subjects who had visited the hospital during that time period were randomly selected for each group. Smoking habits, along with presence of diabetes and hypertension were surveyed, as risk factors were also set as a variable. Of 800 subjects, 206 had history of hypertension, 116 had diabetes, and 133 were current smokers.

The study protocol was reviewed and accepted by Research Ethics Committee, Kyungpook National University (Ethics Reference No. KNUH 2014-07-050-001).

A number of crowns, bridges, implants, and the remaining natural teeth were expressed as mean and standard deviation. Comparisons among the groups or within the groups were performed by independent t-test and one-way ANOVA. Kruskal-Wallis test and Mann-Whitney U test were used for analysis. It was assumed to be statistically significant when $P$ value is below .05 .

To observe the interconnected effect of age, the number of crowns, bridges, implants, and the remaining natural teeth, and smoking, diabetes, and hypertension, a two-way ANOVA test was conducted. Data was assumed to statistically significant when $P$ values were below .05 .

\section{RESULTS}

Differences in the number of crowns, bridges, implants, and the remaining natural teeth related to age were all statistically significant (Table 1). In the case of the remaining natural teeth, the number was gradually decreased as the age increased, so the regularity is remained. But in the case of the crown, bridge and implant, the number was inconstantly distributed. This result indicates that as the aging processes, the teeth with crown or bridge were not saved and implant should be removed. So at some point, the regularity of the crown, bridge and implant disappears (Fig. 1).

Differences in the number of crowns, bridges, implants, and the remaining natural teeth related to hypertension were all statistically insignificant (Table 2). This suggests that the effect of hypertension on the change of oral environment with age is insufficient. 


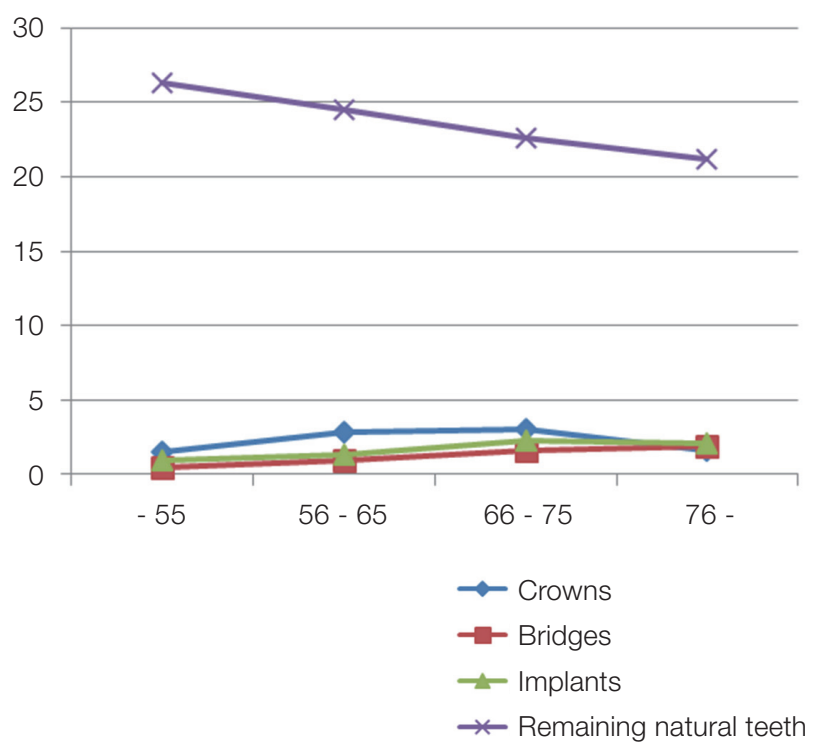

Fig. 1. Graph plotting the variation of crowns, bridges, implants and remaining natural teeth with age. The remaining natural teeth showed a statistically significant decrease with increasing age. The other three variables did not show a constant increase, but showed a statistically significant change.
Data was statistically significant for a 2-way ANOVA test using the number of bridges, age nominal variable, and existence of hypertension as measurement variables (Table 3). However, data was not statistically significant, when a number of crowns, implants, or the remaining natural teeth related to age were used as the variables. A difference in the number of crowns was statistically significant.

Differences in the number of crowns, bridges, and implants related to diabetes were statistically not significant (Table 4). However, a difference in the number of the remaining natural teeth related to diabetes was statistically significant. The relationship with periodontal disease, the sixth complication of diabetes, weakens the resistance of mouth bacteria to invasion and causes periodontal disease due to microvascular complications in the mouth. This is thought to affect the number of remaining teeth.

Data was not statistically significant in a 2-way ANOVA test using the number of bridges, crowns, or implants related to age , and existence of diabetes as measurement variable (Table 5). The reason for a statistically significant change in the number of the remaining natural teeth is considered to be a natural result of an increase in age in addition to the relationship between diabetes and periodontal disease described above.

Table 1. 1-way ANOVA test of number of crowns, bridges, implants, and remaining natural teeth related to age

\begin{tabular}{lrrccc}
\hline & Sum of Square & df & Mean Square & Score & $P$ value \\
\hline Crowns & 412.635 & 3 & 137.545 & 15.342 & $1.035 \mathrm{E}-9$ \\
Bridges & 231.592 & 3 & 77.197 & 52.837 & $3.771 \mathrm{E}-31$ \\
Implants & 235.922 & 3 & 78.641 & 13.711 & $9.995 \mathrm{E}-9$ \\
Remaining natural teeth & $3,226.179$ & 3 & $1,075.393$ & 49.08 & $4.135 \mathrm{E}-29$ \\
\hline
\end{tabular}

Table 2. 1-way ANOVA test of number of crowns, bridges, implants, and remaining natural teeth related to hypertension

\begin{tabular}{lccccc}
\hline & Type III Sum of Square & df & Mean Square & F-score & $P$ value \\
\hline Crowns & 0.028 & 1 & 0.028 & 0.019 & .89 \\
Bridges & 3.384 & 1 & 3.384 & 0.377 & .54 \\
Implants & 11.129 & 1 & 11.129 & 1.942 & .164 \\
Remaining natural teeth & 4.632 & 1 & 4.632 & 0.211 & .646 \\
\hline
\end{tabular}

Table 3. 2-way ANOVA test using number of crown, bridge, implant, remaining natural teeth and age nominal variable and existence of hypertension as measurement variable

\begin{tabular}{lccccc}
\hline & Type III Sum of Square & df & Mean Square & F-score & P value \\
\hline Crowns & 13.068 & 3 & 4.356 & 3.002 & .03 \\
Bridges & 19.815 & 3 & 6.605 & 0.735 & .531 \\
Implants & 18.916 & 3 & 6.305 & 1.1 & .348 \\
Remaining natural teeth & 76.797 & 3 & 25.599 & 1.168 & .321 \\
\hline
\end{tabular}


Table 4. 1-way ANOVA test of number of crowns, bridges, implants, and remaining natural teeth related to diabetes

\begin{tabular}{lccccc}
\hline & Type III Sum of Square & df & Mean Square & F-score & $P$ value \\
\hline Crowns & 0.028 & 1 & 0.028 & 0.019 & .89 \\
Bridges & 5.149 & 1 & 5.149 & 0.575 & .449 \\
Implants & 3.129 & 1 & 3.129 & 0.551 & .458 \\
Remaining natural teeth & 277.98 & 1 & 277.98 & 13.524 & $2.515 \mathrm{E}-4$ \\
\hline
\end{tabular}

Table 5. 2-way ANOVA test using number of crown, bridge, implant, remaining natural teeth and age nominal variable and existence of diabetes as measurement variable

\begin{tabular}{lccccc}
\hline & Type III Sum of Square & df & Mean Square & F-score & $P$ value \\
\hline Crowns & 2.542 & 3 & 0.847 & 0.578 & .629 \\
Bridges & 41.061 & 3 & 13.687 & 1.528 & .206 \\
Implants & 58.167 & 3 & 19.389 & 3.415 & .017 \\
Remaining natural teeth & 559.889 & 3 & 186.63 & 9.08 & $6.491 \mathrm{E}-6$ \\
\hline
\end{tabular}

Table 6. 1-way ANOVA test of number of crowns, bridges, implants, and remaining natural teeth related to smoking

\begin{tabular}{lccccc}
\hline & Type III Sum of Square & df & Mean Square & F-score & $P$ value \\
\hline Crowns & 8.825 & 1 & 8.825 & 6.092 & .014 \\
Bridges & 72.917 & 1 & 72.917 & 8.227 & .004 \\
Implants & 63.215 & 1 & 63.215 & 11.196 & $8.582 \mathrm{E}-4$ \\
Remaining natural teeth & 237.595 & 1 & 237.595 & 11.327 & $8.006 \mathrm{E}-4$ \\
\hline
\end{tabular}

Table 7. 2-way ANOVA test using number of crown, bridge, implant, remaining natural teeth and age nominal variable and existence of smoking as measurement variable

\begin{tabular}{lccccc}
\hline & Type III Sum of Square & df & Mean Square & F-score & $P$ value \\
\hline Crowns & 10.829 & 3 & 3.61 & 2.492 & .059 \\
Bridges & 53.702 & 3 & 17.901 & 2.02 & .11 \\
Implants & 21.338 & 3 & 7.113 & 1.26 & .287 \\
Remaining natural teeth & 513.939 & 3 & 171.313 & 8.167 & $2.329 \mathrm{E}-5$ \\
\hline
\end{tabular}

Differences in the number of crowns and bridges related to smoking were statistically not significant, but differences in the number of implants and remaining natural teeth were statistically significant (Table 6). This suggests that smoking has a greater impact on the oral environment than diabetes or hypertension as previously discussed.

Data was not statistically significant in a 2-way ANOVA test using number of bridges, crowns, or implants related to age, and existence of smoking as measurement variable
(Table 7). However, data was statistically significant when the number of the remaining natural teeth related to age was used as the variable.

This means that the effect of smoking on the survival is significant. Patients with diabetes are $>3$ times more likely to have periodontal disease than those who do not, and patients with diabetes and smoking are $>20$ times more likely to have periodontal disease than those who do not. 


\section{DISCUSSION}

The influences of aging and changes in the oral environment or underlying diseases on the oral environment have been the subjects of many previous studies. Aging is a complex, continuous, and slow process that gradually involves most, if not all, organs of the organism, causing their abnormal functioning in both qualitative and quantitative terms, as well as morphological or structural changes. ${ }^{10}$

Differences from the previous studies are that the sample used in this study is a long-term maintenance periodontal treatment at the Kyungpook National University Dental Hospital and that the parameters for plaque control can be excluded. Because the variables for plaque control can be excluded, we can see the age-related changes occurring under the same oral hygiene condition.

There are many opinions about whether aging affects the oral environment. The aging is closely related to the change of cell, which is the minimum unit of organism. ${ }^{11}$ Due to the aging of cells, the balance between cell division and extinction is broken and cell permeability becomes defective. ${ }^{12}$ The bone density is reduced and the osteoporosis becomes remarkable. In the oral cavity, many changes in chronic diseases are caused, and the cellular immune response to bacterial plaque is reduced. However, the opinion that the gingival recession, alveolar bone loss, and the number of remaining natural teeth are more influenced by environmental factors such as periodontal disease, abusive habits, or missing teeth due to neglect of oral hygiene. As the results of this study show that the variable of aging affects the oral environment, more efforts should be made to manage the oral hygiene with age.

Huttner suggests that aging alone does not cause a significant loss of periodontal attachment in healthy elderly persons. ${ }^{13}$ The effects of human aging on periodontal tissues are based on biomolecular changes of the cells of periodontium that exacerbate bone loss in elderly patients with periodontitis. Although the results of this study were not able to select all healthy subjects, age was found to be a factor affecting the change of oral environment. This is a significant result for the patients who have established oral hygiene through periodontal treatment in this department.

In this study, there was a statistically significant difference in the number of the remaining natural teeth related to diabetes and smoking. As shown in previous studies, diabetes and smoking affect periodontal disease, and age was also a contributing factor. Diabetes has recently been identified as the sixth complication of periodontal disease. ${ }^{14,15}$ In conclusion, the relationship between various systemic diseases and periodontal disease is necessary. A precise study of the risk factors of periodontal disease will also be needed.

Additionally, the number of the remaining natural teeth was statistically significant in most statistical results, but fixed partial dentures and dental implant parameters are not statistically significant because restoration of defects may be possible depending on the treatment plan and the patient's preference. Methods of restoring edentulous areas are gen- erally fixed partial dentures, denture, and implants treatment. Considerations to be made are the patient's preference, the possibility of $\mathrm{Br}$. and denture considering the adjacent teeth, and the existence of alveolar bone suitable for implant placement. Therefore, it is considered that the statistical analysis results are inconsistent with the remaining natural teeth.

Biological or physiological considerations have shown that aging affects the structure of periodontal tissues, the function of the immune response, and the production of gingival associations or plaques. However, the effect of this change on post-treatment response is negligible. Interestingly, recent studies have shown that the elders are more adherent to maintenance therapy than younger patients. ${ }^{16}$ Therefore, it is necessary to preserve natural teeth through proper maintenance, and clinicians and researchers will need more research results related to aging.

\section{CONCLUSION}

Aging is considered to be an important variable affecting the change of oral environment. Among the risk factors, the presence of smoking habit and diabetes is thought to have a great influence on the change of the number of the remaining natural teeth.

\section{ORCID}

Yong Yoon https://orcid.org/0000-0001-9348-1410

Yong-Gun Kim https://orcid.org/0000-0002-2793-7667

Sang-Kyu Lee https:/// orcid.org/0000-0001-5343-701X

Jae-Mok Lee bttps://orcid.org/0000-0002-0291-6114

\section{REFERENCES}

1. Cao J, Venton L, Sakata T, Halloran BP. Expression of RANKL and OPG correlates with age-related bone loss in male C57BL/6 mice. J Bone Miner Res 2003;18:270-7.

2. Cao JJ, Wronski TJ, Iwaniec U, Phleger L, Kurimoto P, Boudignon B, Halloran BP. Aging increases stromal/osteoblastic cell-induced osteoclastogenesis and alters the osteoclast precursor pool in the mouse. J Bone Miner Res 2005;20:1659-68.

3. Benatti BB, Silvério KG, Casati MZ, Sallum EA, Nociti FH Jr. Inflammatory and bone-related genes are modulated by aging in human periodontal ligament cells. Cytokine 2009;46:17681.

4. Yoon DL, Kim YG, Cho JH, Lee SK, Lee JM. Long-term evaluation of teeth and implants during the periodic maintenance in patients with viral liver disease. J Adv Prosthodont 2016;8:321-8.

5. Yoon DL, Kim YG, Cho JH, Lee JM, Lee SK. Long-term evaluations of teeth and dental implants during dental maintenance period. J Adv Prosthodont 2017;9:224-31.

6. Newman MG, Takei HH, Klokkevold PR, Carranza FA. Carranza's Clinical periodontology. 12th ed. St. Louis, MO: Elsevier; 2015. p. 42-6. 
7. Rivaldo EG, Padilha DP, Hugo FN. Alveolar bone loss and aging: a model for the study in mice. J Periodontol 2005;76:196671.

8. The Korean Association of Periodontology Professors. Periodontology. 5th ed. Seoul, Koonja; 2010. p. 55-9.

9. Pauletto NC, Liede K, Nieminen A, Larjava H, Uitto VJ. Effect of cigarette smoking on oral elastase activity in adult periodontitis patients. J Periodontol 2000;71:58-62.

10. Guiglia R, Musciotto A, Compilato D, Procaccini M, Lo Russo L, Ciavarella D, Lo Muzio L, Cannone V, Pepe I, D'Angelo M, Campisi G. Aging and oral health: effects in hard and soft tissues. Curr Pharm Des 2010;16:619-30.

11. Stewart EJ, Madden R, Paul G, Taddei F. Aging and death in an organism that reproduces by morphologically symmetric division. PLoS Biol 2005;3:e45.

12. Ames BN, Shigenaga MK, Hagen TM. Oxidants, antioxidants, and the degenerative diseases of aging. Proc Natl Acad Sci USA 1993;90:7915-22.

13. Huttner EA, Machado DC, de Oliveira RB, Antunes AG, Hebling E. Effects of human aging on periodontal tissues. Spec Care Dentist 2009;29:149-55.

14. Löe H. Periodontal disease. The sixth complication of diabetes mellitus. Diabetes Care 1993;16:329-34.

15. Grossi SG, Genco RJ. Periodontal disease and diabetes mellitus: a two-way relationship. Ann Periodontol 1998;3:51-61.

16. De Visschere LM, Grooten L, Theuniers G, Vanobbergen JN. Oral hygiene of elderly people in long-term care institutions: A cross-sectional study. Gerodontology 2006;23:195-204. 